\title{
Network Threat Analysis based on Vulnerability Relation Model
}

\author{
Zhihong Wang \\ Kunming Vocational and Technical College of Industry, Yunnan 650302, China \\ 1371800697@qq.com
}

\begin{abstract}
A vulnerability relative model based on object time Petri net is proposed for threat evaluation. Combined with Object Oriented technology, the definition of network node object and node class is given, which can help attack information modulation design. By defining the threat of an attack and each index's quantization method, introduce complexity and harmfulness of network attack into the model, and then utilize and improve Dijkstra algorithm to propose non-target oriented and target oriented network threat analysis method.
\end{abstract}

Keywords: Network Security, Attack Model, Threat Evaluation, Attack and Defense Strategy, Petri net

\section{Introduction}

In order to study the relevance between network nodes, the threat of danger, to propagate from the attack path, a potential threat to network vulnerability state made a quantitative evaluation. Most of the existing network threat analysis method has a common characteristic [1-2]: alarm data IDS, COPS and other security tools automatically detected as input [3-4], then by the various modeling methods (such as Bayesian networks, Markov model) analyzed behavior characteristics of attacker, assessed risk status and the probability of the host. It can be concluded that the threat analysis method helped administrator to grasp the current real-time network and attack features of observed, but there are some serious problems:1) by the IDS alarm condition, threat analysis results reasonable relied on the accuracy of the information collection. Reality hacker attack technology is constantly changed and innovation, and some even are to avoid IDS and specially customized, so intrusion detection technology to attack technology updates lag congenital decided defects of the research method. 2) After attacks occurred the analysis of IDS alarm is afterwards Safety Policy. Namely to analysis and response detect after suspicious behavior. For some short time in destructive or new technology, it is difficult to observe the attack behavior cannot be done ahead of active defense, lack of ability to predict attacks. 3) The research method is only limited to the static single evaluating host level, only discussed the risk of this machine holes [5-6].

In order to improve the existing network threat analysis method, make it suitable for the modeling and evaluation of complex network attack system, this paper proposed Network Vulnerability relation model (NVRM) based on object extends time Petri nets [7-8] has more advantages than existing techniques to describe the vulnerability relation and model performance. At the same time, the object oriented technology is introduced, it can help module design of the attack knowledge system. Helped with attack knowledge and network information compiled the $\mathrm{C}++$ code based on component object technology, thus by API interface form to provide each safety components in the active defense system. This paper gives the generation algorithm of network vulnerability relative model, on the basis of algorithms proposed non-target Oriented Threat Analysis (NOTA) and Target Oriented Threat Analysis (TOTA) analysis. 


\section{Vulnerability Relation Model based on Time Petri Nets of Object Extension}

The aim of the course of users (students) is to participate in pre-test learning activities.

\subsection{Definition of the Attack Threat Degree}

[9-10] proposed the concept of network risk, the possibility of successful attacks is by using the weakness and the severity of losses to quantify, this definition is commonly used. This paper proposed the concept of attack threat degree, it reflects security threat level on node object, threat not only considers risk of the attack itself, but also considers the influence of the victim node characteristics.

Definition: The threat of attack Degree (AT). AT is a Two tuple $\langle C, H>, \mathrm{C}$ is attack complexity, used to evaluate the difficulty degree of attack way. $\mathrm{H}$ is attack harmfulness. $H=<S F, N C, N I>$. Where, SF is the effect of attacking on the security properties. Security attributes relate to the confidentiality, integrity and availability. NC is correlation of attack the nodes in the network. Correlation is not only with the topology of the network, is also related to gain access to the trust relationship between the host.

\subsection{Each Index Quantify of the Attack Threat}

Grading standard of attack complexity is proposed in [11], attack complexity $\mathrm{C}$ is according to whether there is ready and available attack tools, public reports and attack step description quantization into 7 grades E1-E7, $C \in[0,1]$. The complexity of an attack mode is lower, more easy to be widely used, the greater threat to network security.

Attack severity $\mathrm{H}$ is by SF, NC and NI decision, where, the SF quantization with vulnerable state node objects in the attack process to consider. Introduced C, I, A threedimensional indicators in the VS set, in combination with the security attributes and fragility give a quantitative method of multi dimension, it is shown in the following Table 1:

Table 1. Multidimensional Quantization Method of the Fragile State and Security Attributes Influence

\begin{tabular}{|l|l|l|l|}
\hline \multicolumn{1}{|c|}{$\begin{array}{c}\text { Security } \\
\text { Attributes }\end{array}$} & Confidentiality(C) & Integrity(I) & Availability (A) \\
\hline Vccess & 0.1 & --- & 0.2 \\
\hline User & 0.5 & 0.3 & 0.4 \\
\hline Root & 1 & 0.8 & 1 \\
\hline Dos & --- & --- & 0.8 \\
\hline Info-leak & 0.8 & 0.1 & 0.2 \\
\hline Controlled & 0.2 & 0.6 & 0.6 \\
\hline
\end{tabular}

Node correlation reflects the network correlation of the object node. The correlation refers not only to the physical link layer. Relations are also included in the IP layer and the application layer service or application. Correlation of a node object is higher, the correlation is stronger, more easily is be exploited by attackers, and therefore threat is the greater. Node correlation is calculated according to the node object relationship R. The formula is as follows:

$$
N C\left(O_{i}\right)=\frac{n u m_{T R}\left(O_{i}\right)+\operatorname{num}_{A R}\left(O_{i}\right)}{\sum_{O_{j} \in U}^{N} n u m_{T R}\left(O_{j}\right)+\operatorname{num}_{A R}\left(O_{j}\right)}
$$


Where, num $_{T R / A R}\left(O_{i}\right)$ describes the relationship of each node object and the number of records. The association degree of $O_{i}$ is calculated by the correlation ratio of total network relationship number. Due to the inevitable physical connection $P R$ between host trust relationship and access relationship the host, network attacks mainly used relationship is $T R$ and $A R$. There is no consideration of $P R$.

Node important degree is measured by $P r$ and $C r$, [12-13] proposed the division of assets value level, according to the important degree of a node and host key levels of node is defined by Table 2, Table 3. Table 2 and Table 3 showed important classification description and the corresponding quantitative value.

Table 2. Categories of Node Importance

\begin{tabular}{|l|l|l|l|l|}
\hline Host & Server & Firewall & \multicolumn{1}{|c|}{ Router } & IDS \\
\hline 0.4 & 0.8 & 0.5 & 0.7 & 0.2 \\
\hline
\end{tabular}

Table 3. The Main Key Classification

\begin{tabular}{|c|c|c|c|}
\hline Classification & Authority & $\begin{array}{l}\text { The application or } \\
\text { service }\end{array}$ & Data \\
\hline 0.1 & $\begin{array}{l}\text { Only has the } \\
\text { permissions on the } \\
\text { device resources }\end{array}$ & $\begin{array}{l}\text { Run the application } \\
\text { and service in general, } \\
\text { have little effect }\end{array}$ & $\begin{array}{l}\text { Common file users or } \\
\text { enterprises and non- } \\
\text { sensitive data }\end{array}$ \\
\hline 0.5 & $\begin{array}{l}\text { To the authority } \\
\text { without verifying } \\
\text { login other host and } \\
\begin{array}{ll}\text { access to important } \\
\text { data }\end{array}\end{array}$ & $\begin{array}{l}\text { Run application of } \\
\text { important, have a } \\
\text { certain effect on the } \\
\text { node and network } \\
\text { function }\end{array}$ & $\begin{array}{l}\text { Important information } \\
\text { users or enterprises } \\
\text { and sensitive data }\end{array}$ \\
\hline 0.8 & $\begin{array}{lr}\text { To implement } & \text { the } \\
\text { allocation } & \text { and } \\
\text { management } & \text { by } \\
\text { firewall, server node } \\
\text { to the authority }\end{array}$ & $\begin{array}{l}\text { Run important } \\
\text { applications } \\
\text { services, have a great } \\
\text { impact on the network }\end{array}$ & $\begin{array}{l}\text { The user or enterprise } \\
\text { extremely important } \\
\text { data and confidential } \\
\text { data }\end{array}$ \\
\hline
\end{tabular}

In Table 3 main key indicators is determined according to the actual attack level, when the three indicators at different levels to meet, get maximum values.

According to the quantification method above, can calculate the threat of loophole attack behavior, it is calculated as follows:

$$
A T\left(M\left(p_{i}\right)\right)=\alpha *(1-C)+\beta *\left(\alpha_{1} *\left(P_{c}^{*} C+P_{i}^{*} I+P_{a}^{*} A\right)+a_{2} * N C+a_{3} *\left(b_{1} * P r+b_{2} * C r\right)\right)
$$

Formula (2) can be calculated threat according to the method of $M$ to network vulnerable state $p_{i}, \alpha, \beta, \alpha_{1}, \alpha_{2}, \alpha_{3}, b_{1}, b_{2}, b_{3}, P_{c}, P_{i}, P_{a}$ are weight of each index, according to the actual situation evaluated focus selection. In order to vulnerability assessment of network nodes and the optimal attack strategy to lay the foundation.

\section{Analysis Method of Network Threats based on Vulnerability Relation Model}

In different circumstances, analysis of network administrators to threats of accuracy is often different. Sometimes, a few focus nodes carried out safety assessment, sometimes, needs to assess the overall network. For different needs, the model should have certain versatility. Therefore, proposed non-target oriented and target oriented network threat analysis method based on network vulnerability relation model 


\subsection{Network Threats Analysis of Non-target Oriented}

Non-target oriented refers to the network any node on the fragile state are likely to be the goal of the attacker, in this case, the network administrator needs to obtain threat value of each fragile state, in order to evaluate nodes and weak state threatened the largest in complete attack information network. Dijkstra algorithm is the classical shortest path algorithm, used to calculate the shortest path from a single source to all other nodes. Its characteristics are suitable for target oriented solving the problem of threat. But the application of Dijkstra algorithm in graph theory is only a single path based on node, network vulnerability correlation model is modeling by Petri net, excitation transition place between nodes can be more than one, that model the attacker can adopt different attack methods to achieve the same objective on the same node, so needs the Dijkstra algorithm to improve. Here are non-target oriented network threat degree calculation methods:

Algorithm: NOTA (ILARM, ini_place)

\section{Input: ILARM}

Output: Each database node threat $\xi_{i}$ and AT node object threat $A T\left(O_{j}\right)$

1 Build three-dimensional array $C[n][n][v]$ stored change complexity $\lambda$ of each database node in NVRM model. $C[i][j][k]$ shows complexity of attack $t_{k}$ from $p_{i}$ to $p_{j}$. Builds array $H[m][n]$ storage hazard $\mu . H[k][j]$ shows hazard from $t_{k}$ to $p_{j}$.

2 Initialize: $\mathrm{P}$ is network library set, $S=\left\{p_{0}\right\}, \xi_{0}=0$ network library.

3 For $\left(\right.$ all $p_{i} \in P-S$ )

$4 A T\left(p_{i}\right)=\max \left(\alpha *(1-C[0][i][0])+\beta * H[0][i], \ldots, \alpha^{*}(1-C[0][i][k])+\beta * H[k][i]\right)$

5 Generated tok $\left(p_{i}\right), \operatorname{tok}\left(p_{i}\right) \cdot A T=A T\left(p_{i}\right)$.rout $=p_{0}, t_{h}, p_{i}$

6 For $(i=0 ; i<n ; i++)$

$7 \xi_{k}=\max \left\{\operatorname{Tok}\left(p_{i} \cdot A T \mid \forall p_{i} \in P-S\right\}\right.$

$8 f\left(\xi_{k}==0\right)$

9 Break

$10 S=S \cup\left\{p_{k}\right\}$

11 For (all $p_{j} \in P-S$ )

12 For $(h=0 ; h<v ; h++)$

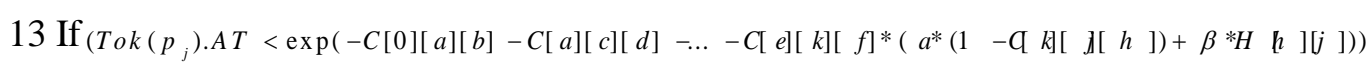

$14 \operatorname{Tok}\left(p_{j}\right) \cdot A T=A T_{k}$

$\left.15 \operatorname{Tok}\left(p_{j}\right) \cdot \operatorname{rout}=\operatorname{Tok}\left(p_{k}\right) \cdot \operatorname{rout}+" t_{h}, p_{j} "\right)$

16 If $\left(\operatorname{Tok}\left(p_{j}\right) \cdot A T=A T_{k}\right)$

$17\left\{\right.$ Create a new token $\operatorname{Tok}_{\text {new }}\left(p_{j}\right)$ 
$18 \operatorname{Tok}_{\text {new }}\left(p_{j}\right) \cdot A T=A T_{k} ; \operatorname{Tok}_{\text {new }}\left(p_{j}\right) \cdot \operatorname{rout}=\operatorname{Tok}\left(p_{k}\right) \cdot$ rout $\left.+t_{h}, p_{j} "\right\}$

$19 \xi_{j}=\operatorname{Tok}\left(p_{j}\right) \cdot A T$

20 For $(j=1 ; j \leq N ; j++)$

21 For $\left(A l l \quad p_{i} \in O_{j}\right)$

$22 A T\left(O_{j}\right)+=\xi_{i}$

23 Output $\xi_{i}, A T\left(O_{j}\right)$

\subsection{Network Threats Analyses of Target Oriented}

Target oriented refers to the network administrator during the network threat analysis has a certain focus, evaluates the degree of threat and the status of certain nodes according to input target. These nodes and the state are to be evaluated with the target has a direct or indirect relationship. In the 3.1 section, by NOTA (ILARM, ini_place) algorithm can obtain the optimal token $\operatorname{Tok}\left(p_{j}\right)$ in network, which records the corresponding threat degree value and path information of experience. Used to reach recording of the target state tokens, target oriented network threats is analyzed. This attack can be one target, there can be multiple target. Analysis steps are as follows:

Step1: Input target database $p_{j}$. Remove the corresponding token $T o k\left(p_{j}\right)$ from $p_{j}$, the token rout record sequence of the biggest threat propagation path. The $p_{j}$ threat obtained directly by $\operatorname{Tok}\left(p_{j}\right) . A T$

Step2: In the $\operatorname{Tok}\left(p_{j}\right)$.rout . Rout find the path by all the library $\left\{p_{0}, p_{a}, p_{c}, \ldots, p_{j}\right\}$ and remove the corresponding token $\left\{\operatorname{Tok}\left(p_{a}\right), \operatorname{Tok}\left(p_{c}\right), \ldots\right\}$ except the initial $p_{0}$ and the target base $p_{j}$, with their target the $A T$ values stored in the array $A T[]$, array subscript represents the number, array element stores threat degree value.

Step3: If there is no input target database, output $A T$ [] obtains all threat degree associated with target. Otherwise, continue to operate the first two steps until no input.

Step4: Calculated node degree of threat $A T\left(O_{j}\right)$. Value of $A T\left(O_{j}\right)$ can obtain belongs to the same node object by the key of additive in $A T[]$.

\section{Experimental Design and Discussion}

The paper established attack scenarios to show the establishment of network vulnerability relation model and analysis method of the database object threat. The environment consists of three parts: network Internet, DMZ isolation zone and the internal LAN. Firewall is running workstation of the Linux operating system. IP1 is an intrusion detection equipment, is connected between the firewall and DMZ switch. DMZ contains two hosts: IP2 is an IIS Web server, IP3 is running the SSH server on REDHAT Linux, provide the FTP service. The internal LAN, IP4 is installed the firewall management software, IP4 can directly control the firewall. IP5 is the user machine. IP6 runs key database, and stores enterprise confidential data. Firewall enable external host can only access the host of the DMZ regions, cannot directly access the internal LAN; while the host of the DMZ region can access the internal LAN. Assuming that hacker is in Internet, to launch an attack on DMZ and Intranet hosts and servers. If an attacker outside the network used HTTP to directly attack buffer overflow vulnerability of the Web server, will be NIDS system to identify and block. 


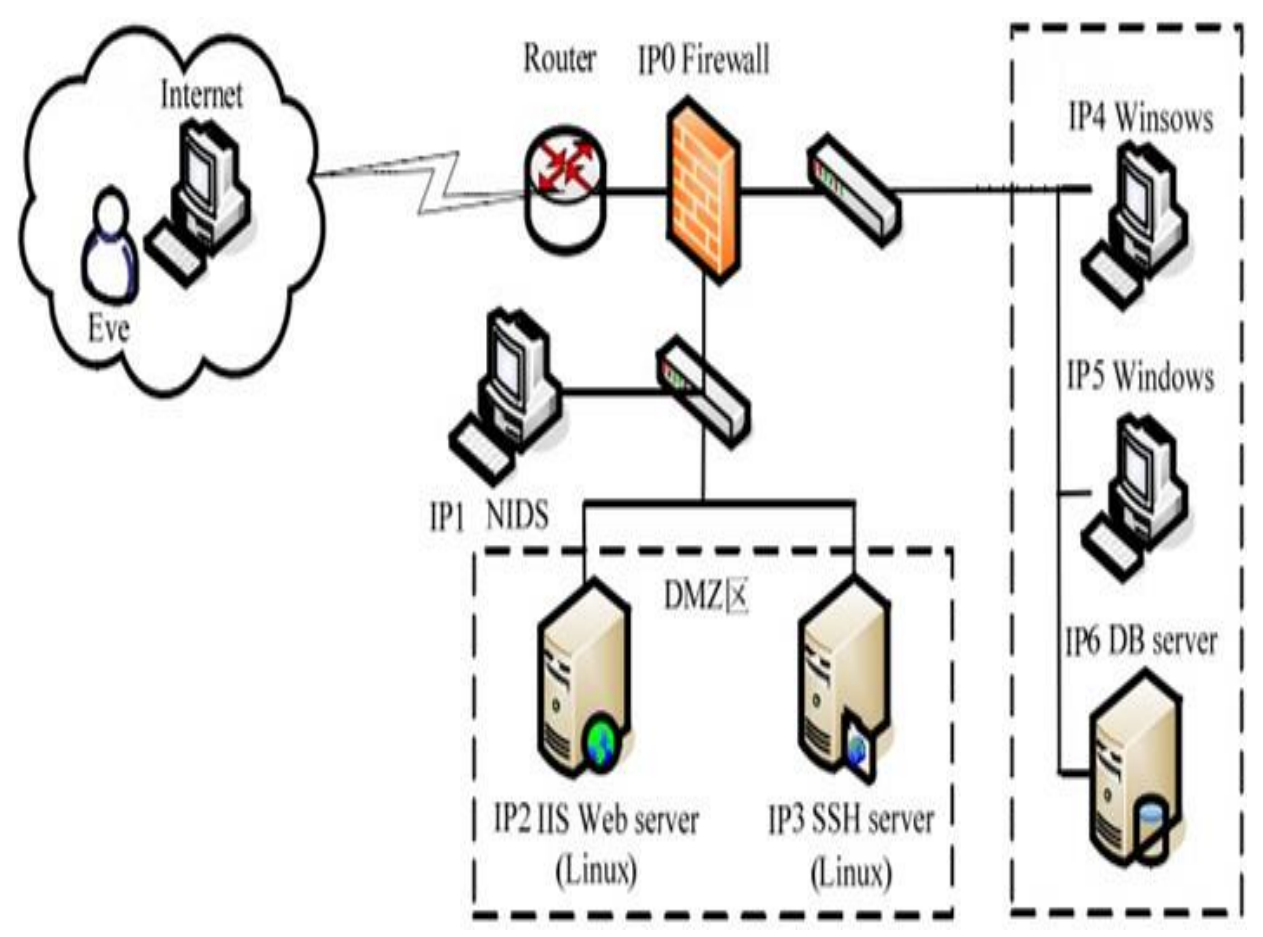

Figure 2. The Network Topology Map

In the test environment, each node in the network, Eve, Router, Firewall and IP1-IP6 created an instance of an object, encapsulating the information in the object:

In Table 2, static attribute of each node object only lists the vulnerability information on the nodes. Other basic information is omitted here. Fragile state initial dynamic property is false. The state has not occurred. Object association is recorded to access services, port and host between user nodes. According to the NVRM algorithm to establish vulnerability relative model, it is shown in Figure 3 and Figure 4.

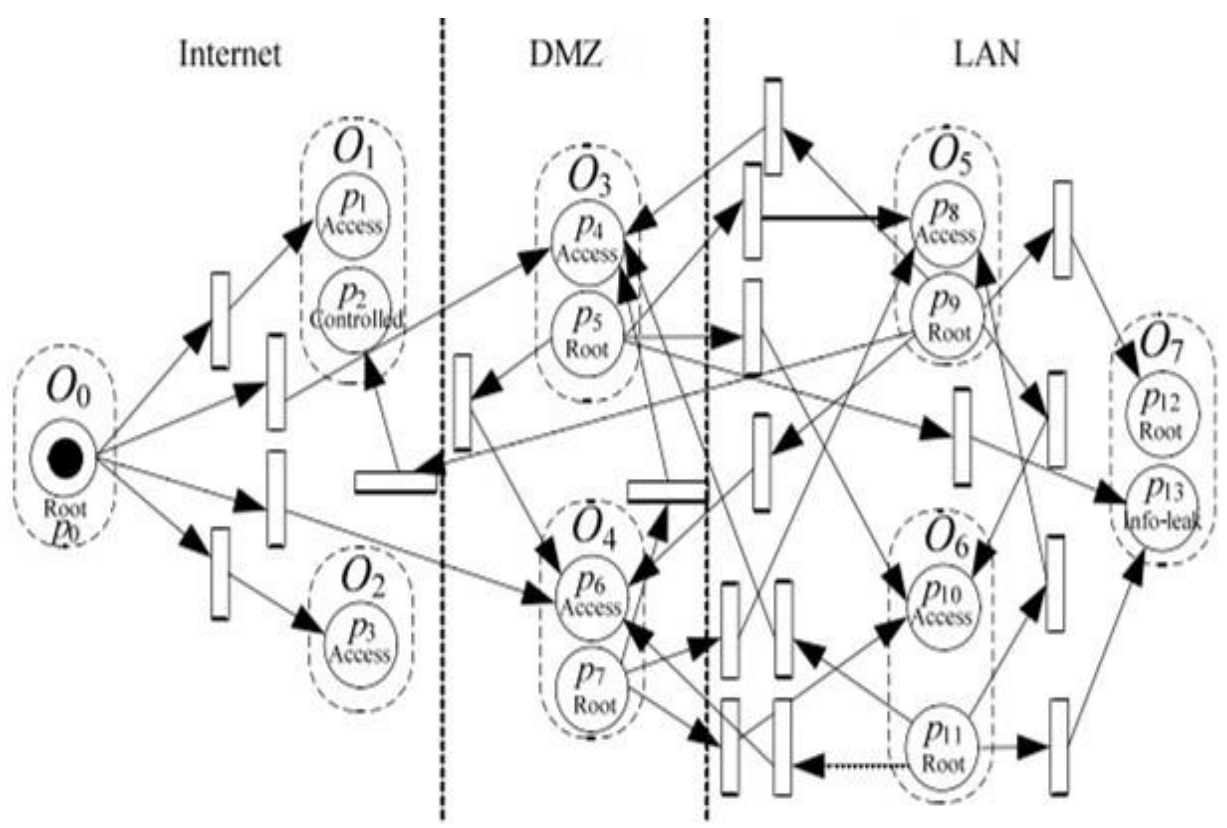

Figure 3. Legitimate Access Relationship of the Node Object (LARM) 
Table 1. Establishment of Node Object

\begin{tabular}{|c|c|c|c|}
\hline $\begin{array}{l}\text { Node } \\
\text { object }\end{array}$ & $\begin{array}{l}\text { Static } \\
\text { properties }\end{array}$ & $\begin{array}{l}\text { Dynamic } \\
\text { attributes }\end{array}$ & Object relationship \\
\hline$O_{0}(E v e)$ & Null & Null & $\begin{array}{l}\operatorname{Access}\left(O_{0}, \text { user, } O_{1}, \text { port }(23), \text { Root, Access }\right) \\
\operatorname{Access}\left(O_{0}, \text { user, } O_{2}, \text { port }(7,13), \text { Root }, \text { Access }\right) \\
\operatorname{Access}\left(O_{0}, \text { user, } O_{3}, w w w, \text { Root }, \text { Access }\right) \\
\operatorname{Access}\left(O_{0}, \text { user, } O_{4}, \text { ftp, Root, Access }\right)\end{array}$ \\
\hline$O_{1}(I P 0)$ & $\begin{array}{l}12918, \\
\text { RedHat } \\
\text { Linux } \\
\text { telnetOverf } \\
\text { low (Root/ } \\
\text { Controlled } \\
\text { ) }\end{array}$ & $\begin{array}{l}\mathrm{VS}= \\
\mathrm{FALSE}\end{array}$ & Null \\
\hline $\mathrm{O}_{2}(I P 1)$ & $\begin{array}{l}38479, \text { net_- } \\
\text { ule(Linux) } \\
\text { Remote } \\
\text { Denial of } \\
\text { service } \\
(\text { DoS) }\end{array}$ & $\begin{array}{l}\mathrm{VS}= \\
\mathrm{FALSE}\end{array}$ & Null \\
\hline$O_{3}(I P 2)$ & $\begin{array}{l}4855, \quad \text { IIS } \\
\text { buffer } \\
\text { overflow( } \\
\text { Root/DoS) }\end{array}$ & $\begin{array}{l}\mathrm{VS}= \\
\mathrm{FALSE}\end{array}$ & $\begin{array}{l}\operatorname{Access}\left(\mathrm{O}_{3}, \text { user, } \mathrm{O}_{41}, \text { ftp }, \text { Root }, \text { Access }\right) \\
\operatorname{Access}\left(\mathrm{O}_{3}, \text { user, } \mathrm{O}_{5}, \text { port }(445), \text { Root }, \text { Access }\right) \\
\operatorname{Access}\left(\mathrm{O}_{3}, \text { user }, \mathrm{O}_{6}, \text { port }(135), \text { Root }, \text { Access }\right) \\
\operatorname{Access}\left(\mathrm{O}_{3}, w w w, \mathrm{O}_{7}, \text { oracle }, \text { Root }, \text { Info-leak }\right)\end{array}$ \\
\hline $\mathrm{O}_{4}(I P 3)$ & $\begin{array}{l}8628, \\
\text { OpenSSH } \\
3.3 \\
\text { bufferOver } \\
\text { flow(Root) }\end{array}$ & $\begin{array}{l}\text { VS }= \\
\text { FALSE }\end{array}$ & $\begin{array}{l}\operatorname{Access}\left(\mathrm{O}_{4}, \text { user, } O_{3}, w w w, \text { Root, Access }\right) \\
\operatorname{Access}\left(O_{4}, \text { user, } O_{5}, \operatorname{port}(445), \text { Root, Access }\right) \\
\operatorname{Access}\left(\mathrm{O}_{4}, \text { user }, O_{6}, \operatorname{port}(135), \text { Root, Access }\right)\end{array}$ \\
\hline$O_{5}(I P 4)$ & $\begin{array}{l}8152 \\
\text { SMB } \\
\text { remote } \\
\text { data } \\
\text { destruction } \\
\text { of } \\
\text { vulnerabili } \\
\text { ty (Root) }\end{array}$ & $\begin{array}{l}\text { VS= } \\
\text { FALSE }\end{array}$ & 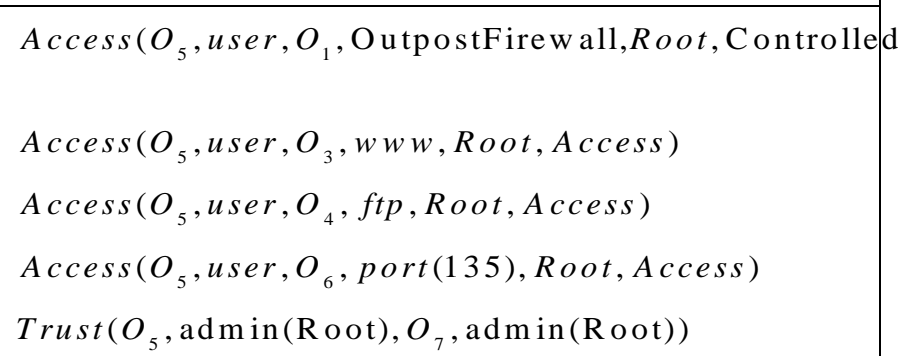 \\
\hline $\mathrm{O}_{6}(I P 5)$ & $\begin{array}{l}31874, \text { the } \\
\text { Windows } \\
\text { Server } \\
\text { service } \\
\text { remote } \\
\text { Rpc } \\
\text { overflow } \\
\text { (Root) }\end{array}$ & $\begin{array}{l}\mathrm{VS}= \\
\mathrm{FALSE}\end{array}$ & $\begin{array}{l}\operatorname{Access}\left(\mathrm{O}_{6}, \text { user, } \mathrm{O}_{3}, w w w, \text { Root }, \text { Access }\right) \\
\operatorname{Access}\left(\mathrm{O}_{6}, \text { user, } \mathrm{O}_{4}, \text { ftp, Root, Access }\right) \\
\operatorname{Access}\left(\mathrm{O}_{6}, \text { user, } \mathrm{O}_{5}, \text { port }(445), \text { Root }, \text { Access }\right) \\
\operatorname{Access}\left(\mathrm{O}_{6}, \text { user }, \mathrm{O}_{7}, \text { oracle }, \text { Root }, \text { Info-leak }\right)\end{array}$ \\
\hline
\end{tabular}




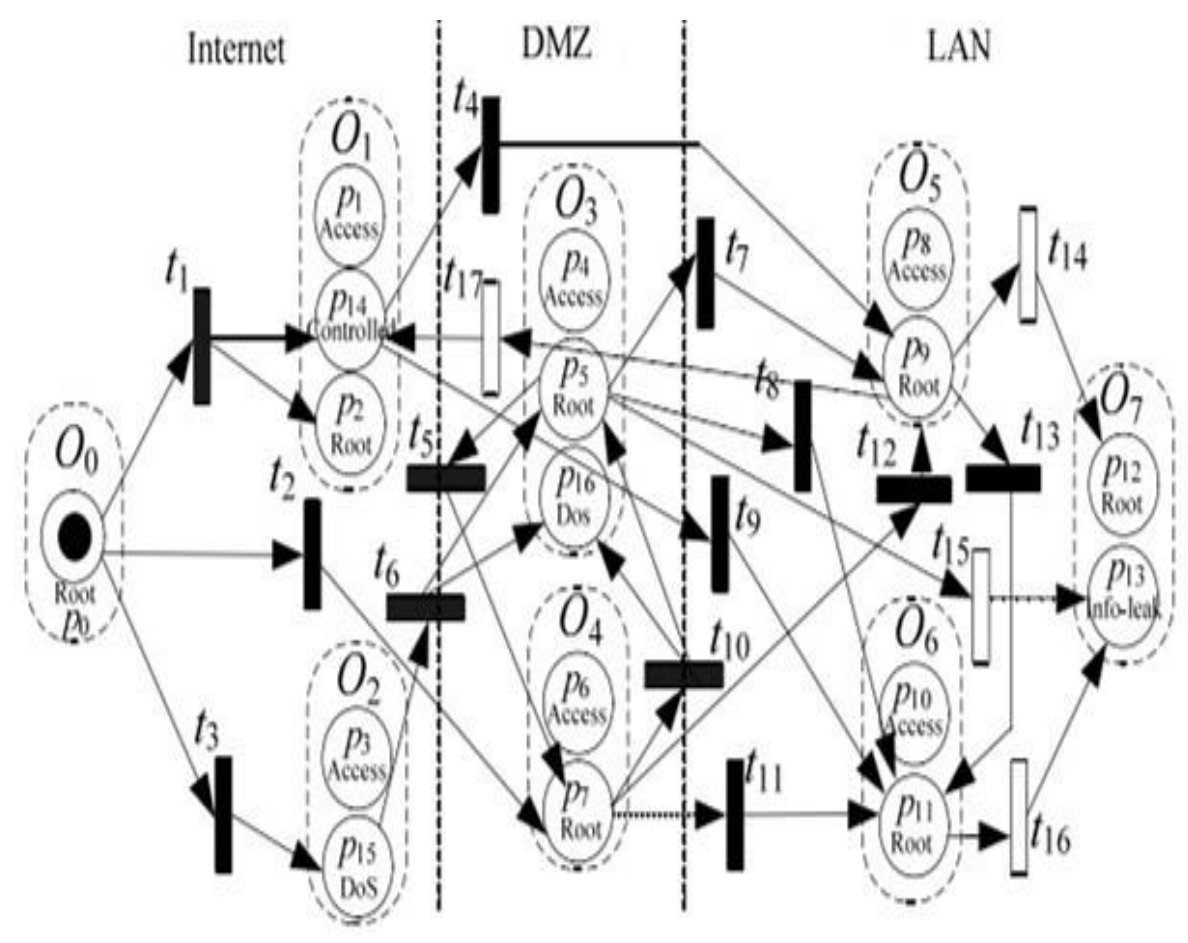

Figure 4. Illegal Attack Relationship of the Node Object

Figure6 hollow change showed Legal relationship between the object, solid change showed malicious behavior of object vulnerabilities. According to the quantization method in section 2.2 and formula (2), which can calculate the change complexity $\lambda_{h}$ and attack the harm degree $\mu_{h q}$. According to the correlation degree of each node object of the formula

$N C\left(O_{1}\right)=0.057, N C\left(O_{2}\right)=0.029 N C\left(O_{3}\right)=0.229, N C\left(O_{4}\right)=0.2, N C\left(O_{5}\right)=0.2$

$N C\left(O_{6}\right)=0.2, N C\left(O_{7}\right)=0.086$.In the paper, threat degree of each index weights is equal, coefficient is following in formula (2): $\alpha=\beta=1 / 2, a_{1}=a_{2}=a_{3}=1 / 3, b_{1}=b_{2}=1 / 2, P_{c}=P_{i}=P_{a}=1 / 3$, combining security attributes and node important values can be obtained as table2:

Table 2. ILARM Change and Output Arc on the Complexity and the Degree of Harm

\begin{tabular}{|l|l|l|l|l|l|l|l|l|l|l|}
\hline$\lambda_{1}=\lambda_{2}$ & $\lambda_{3}$ & $\lambda_{4}$ & $\lambda_{5}$ & $\lambda_{6}$ & $\lambda_{7}$ & $\lambda_{8}=\lambda_{9}$ & $\lambda_{10}$ & $\lambda_{11}=\lambda_{12}$ & $\lambda_{13}$ & $\lambda_{14}$ \\
\hline 0.3 & 0.5 & 0.7 & 0.3 & 0.1 & 0.7 & 0.7 & 0.1 & 0.7 & 0.7 & 0.1 \\
\hline$\lambda_{15}=\lambda_{16}$ & $\lambda_{17}$ & $\mu_{12}$ & $\mu_{1-14}$ & $\mu_{27}$ & $\mu_{3-15}$ & $\mu_{49}$ & $\mu_{57}$ & $\mu_{65}$ & $\mu_{6-16}$ & $\mu_{79}$ \\
\hline 0.2 & 0.1 & 0.547 & 0.391 & 0.594 & 0.149 & 0.578 & 0.594 & 0.604 & 0.382 & 0.578 \\
\hline$\mu_{8-11}$ & $\mu_{9-11}$ & $\mu_{10-15}$ & $\mu_{10-16}$ & $\mu_{11-11}$ & $\mu_{12-9}$ & $\mu_{13-11}$ & $\mu_{14-12}$ & $\mu_{15-13}$ & $\mu_{16-13}$ & $\mu_{17-14}$ \\
\hline 1.53 & 1.8 & 3 & 2.6 & 1.6 & 0.93 & 2.02 & 1 & 3.54 & 5 & 5.3 \\
\hline 0.461 & 0.461 & 0.604 & 0.382 & 0.461 & 0.578 & 0.461 & 0.606 & 0.418 & 0.418 & 0.391 \\
\hline
\end{tabular}

Table 2 is the establishment of a test network vulnerability relative model. The following will be ILARM as non-target threat analysis algorithm input, calculated node threat of various database. 
In the case of not setting goals, NOTA algorithm will be generated from $O_{0} \cdot p_{0}$ to $O_{1}-O_{7}$ threat of the path of maximum, and the path is corresponding to the threat degree value. It is shown in table3. In this paper, by calling $N O T A\left(\operatorname{ILARM}, O_{0} . p_{0}\right)$ can be a node object threat and reach the optimal path of each place.

In Figure 4, attackers can directly attack object IP0, IP1 and IP3, due to the presence of NIDS. Buffer overflow vulnerabilities cannot be directly attacked the IP2.

IP3 provides services to internal and external users, association in the network is large and important. The attacker can obtain root permission by holes 8628 , therefore in table 3 $p_{7}$ is Threat maximum. Security strategy of firewall IP0 is to deploy the internal LAN external shielding, once lost, the attacker can direct attack intranet host and service device, so the IP0 is very important in the security status, but the network connection of IP0 is smaller, so the threat degree is slightly lower than IP3. IP1 (IDS) function and role in the network are relatively weak, so the threat degree is low. The fragile state of the rest of the objects needed multistage attack to achieve, attack complexity increases gradually with the path length increased, threatening decreased. On IP2 the loopholes, existing can be directly attack tool IIS5HACK, the attack complexity is very low, and the attack can also lead to two fragile state, each state has a greater impact on the network function, so the total threat of node $O_{3}$ is high. Notably, $p_{9}, p_{11}, p_{12}$ has two threat degree maximum paths, because the complexity $\lambda_{1}, \lambda_{2}$ of change $t_{1}, t_{2}$ is the same. Used telnet buffer overflow vulnerabilities of IP0 to make the attacker can modify the security policy, IP4 and IP5 can be directly attacked the network, at the same time, the attacker can attack the IP3 server, used it to attack IP4 and IP5 by Intranet access relation, in the 12918 and 8628 attack complexity is similar, the two attack strategies are optimal strategy.

Table 3. Non-target Oriented of All Nodes Threat

\begin{tabular}{|l|l|l|l|l|}
\hline \multicolumn{2}{|c|}{$\mathrm{O}_{1}(\mathrm{IP} 0)$} & $\mathrm{O}_{2}(\mathrm{IP} 1)$ & \multicolumn{2}{c|}{$\mathrm{O}_{3}(\mathrm{IP} 2)$} \\
\hline$p_{2}$ & $p_{14}$ & $p_{15}$ & $p_{5}$ & $p_{16}$ \\
\hline$\xi_{2}=0.623$ & $\xi_{14}=0.546$ & $\xi_{15}=0.324$ & $\xi_{5}=0.557$ & $\xi_{16}=0.475$ \\
\hline$p_{0}, t_{1}, p_{2}$ & $p_{0}, t_{1}, p_{14}$ & $p_{0}, t_{3}, p_{15}$ & $p_{0}, t_{2}, p_{7}, t_{10}, p_{5}$ & $p_{0}, t_{2}, p_{7}, t_{10}, p_{16}$ \\
\hline \multicolumn{2}{|c|}{$A T\left(O_{1}\right)=1.169$} & $A T\left(O_{2}\right)=0.324$ & \multicolumn{2}{|c|}{$A T\left(O_{3}\right)=1.032$} \\
\hline $\mathrm{O}_{4}(\mathrm{IP} 3)$ & $\mathrm{O}_{5}(\mathrm{IP} 4)$ & $\mathrm{O}_{6}(\mathrm{IP} 5)$ & $\mathrm{O}_{7}(\mathrm{IP} 6)$ & $p_{13}$ \\
\hline$p_{7}$ & $p_{9}$ & $p_{11}$ & \multicolumn{1}{|c|}{$p_{12}$} & $\xi_{7}=0.408$ \\
\hline$\xi_{7}=0.647$ & $\xi_{9}=0.325$ & $\xi_{11}=0.282$ & $\xi_{7}=0.277$ & $p_{0}, t_{2}, p_{7}, t_{10}, p_{5}, t_{15}, p_{13}$ \\
\hline$p_{0}, t_{2}, p_{7}$ & $p_{0}, t_{2}, p_{14}, t_{4}, p_{9}$ & $p_{0}, t_{2}, p_{14}, t_{9}, p_{11}$ & $p_{0}, t_{1}, p_{14}, t_{4}, p_{9}, t_{14}, p_{12}$ & \\
\hline$A T\left(O_{4}\right)=$ & $0_{0.67\left(O_{5}\right)=0.325} A T\left(O_{6}\right)=0.282$ & $p_{0}, t_{2}, p_{7}, t_{12}, p_{9}, t_{14}, p_{12}$ & \\
\hline
\end{tabular}

In the target oriented, assuming $p_{9}$ and $p_{13}$ is as the target to achieve optimal path are $p_{0}, t_{1}, p_{14}, t_{4}, p_{9} ; p_{0}, t_{2}, p_{7}, t_{12}, p_{9}$ and $p_{0}, t_{2}, p_{7}, t_{10}, p_{5}, t_{15}, p_{13}$. The fragile state $p_{5}, p_{7}, p_{9}, p_{13}, p_{14}$ does not contribute for the attack target, Threat of the node object is
$A T\left(O_{1}\right)=0.546$
$A T\left(O_{2}\right)=0$
$A T\left(O_{3}\right)=0.557$
$A T\left(O_{4}\right)=0.647$ 
$A T\left(O_{5}\right)=0.325, A T\left(O_{6}\right)=0, A T\left(O_{7}\right)=0.408$. To attack the goal, not involve the threat degree of node is 0 .

According to Figure 3 and the threat analysis, object IP3 and IP0 are unable to external network mask, so the maximum degree of risk $p_{7}, p_{12}$, by repairing their vulnerability is to reduce the best strategy of the threat. P4 and IP5 host of intranet is threatened the main reason is DMZ region Intranet access. Although the IP6 database does not exist loophole, Therefore, protection of IP6 is to control DMZ region on intranet access permissions, and repairs IP5 and IP6 vulnerabilities.

[14] used the attack graph model to establish a model for the 5 network device, produced 54 network nodes without limiting the number and success rate of attack case. The paper test network has 7 important equipment, vulnerability relation model has harmful node only 10, compared to the attack graph model, modeling method of NVRM greatly reduces the number nodes of network state without limiting the model ability situation. In the threat analysis, the paper proposed goal-oriented and non-goal-oriented analytical methods are more comprehensive and reasonable than $[6,8]$

\section{Conclusion}

In order to network nodes threatening of comprehensive consideration, this paper establishes the threat degree evaluation index system and gives corresponding quantification methods, by using the extended time Petri net is achieve relation model of network vulnerability, the defense is more accurately to grasp the network attack behavior and step. The model carries to decide attack complexity and attack severity attribute. Finally, proposed the method of threat assessment for goal-oriented and non-goal-oriented

\section{References}

[1] A. Arnes, F. Valeur and G. Vigna, "Using hidden Markov models to evaluate the risk of intrusions", Proceedings of the RAID’06, Hamburg, Germany, (2006), pp. 145-164.

[2] E. Jonsson and T. Olovsson, "A quantitative model of the security intrusion process based on attacker behavior", IEEE Transactions on Software Engineering, vol. 23, no. 4, (1997), pp. 235-24.

[3] K. Goseva-Popstojanova, F. Wang, R. Wang and Vaidyanathan, "Characterizing intrusion tolerant systems using a state transition model", In DARPA Information Survivability Conference and Exposition, (2001), pp. 211-221.

[4] V. Mehta, C. Bartzis and H. Zhu, "Ranking attack graphs", Proceedings of the International Symposium on the Recent Advances in Intrusion Detection (RAID 2006), Springer-Verlag, (2006), pp. 127-144.

[5] X. Chen, Q. Zheng and X. Guan, "Evaluation method of quantitative hierarchical network security threat", Journal of software, vol. 17, no. 4, (2006), pp. 885-897.

[6] T. Li, "Detection of network security risk based on immunity", China Science (series E - Information Science), vol. 35, no. 8, (2005), pp. 798-816.

[7] W. Li, J. Lei and J. Dong, "An optimization method for real time network security risk quantification", Chinese Journal of computers, vol. 32, no. 4, (2009), pp. 794-803.

[8] Y. Zhang, B. Fang and Y. Chi, "Research on correlation in network risk assessment of network nodes", Chinese Journal of computers, vol. 30, no. 2, (2007), pp. 235-240.

[9] Y. Zhang, B. Fang and Y. Chi, "Risk propagation model for evaluation of network information system", Journal of software, (2007), vol. 18, no. 1, pp. 137-145.

[10] G. Biswas, K. A. Debelak and K. Kawamura, "Applications of qualitative modeling to knowledge-based risk assessment studies", Proceedings of the 2nd Conference, on Industrial and Engineering Applications of Artificial Intelligence and Expert Systems, Tullahoma: ACM Press, (1989), pp. 92101.

[11] Y. Zhang, X. Yun and M. Hu, "Research on vulnerability taxonomy with multidimensional quantitative ttribute privilege escalating based", Journal of communication, vol. 25, no. 7, (2004), pp. 107-114.

[12] B. Liu, X. Wang and Y. Chi, "Research and practice of network security evaluation method", Computer Engineering, vol. 33, no. 16, (2007), pp. 133-138.

[13] X. Liu, N. Ma and D. Xiao, "Vulnerability assessment of risk theory based network", Journal of Wuhan University of Technology, vol. 18, no. 9, (2009), pp. 39-42. 
[14] J. E. Strutt, J. D. Patrick and N. D. E. Custance, "A risk assessment methodology for security advisors", Proceedings of the 29th IEEE Annual Int'l Carnahan Conference on Security Technology, Sanderstead: IEEE Computer Society Press, (1995), pp. 225-229.

\section{Author}

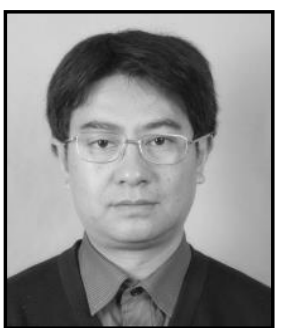

Zhihong Wang, $\mathrm{He}$ is currently an associate Professor in Kunming Vocational and Technical College of Industry. His research interests include Development of application systems for office automation, automated micro-grid troubleshooting for electric power systems. 
International Journal of Security and Its Applications

Vol.9, No.1 (2015) 